\title{
Convalescent plasma treatment for patients of 80 years and older with COVID-19 pneumonia
}

\author{
Iñigo Romon ${ }^{*}$, Juan J. Dominguez-Garcia', Jose L. Arroyo ${ }^{2}$, Borja Suberviola ${ }^{3}$, Itxasne Cabezón ${ }^{4}$, Beatriz Abascal ${ }^{5}$, \\ Cristina Baldeón ${ }^{6}$, Amalia Cuestaํ, Raquel Portilla ${ }^{8}$, Elena Casuso ${ }^{9}$, Enrique Ocio ${ }^{1}$ and Montserrat Briz ${ }^{1}$
}

\begin{abstract}
Background: Older patients, frequently with multiple comorbidities, have a high mortality from COVID-19 infection. Convalescent plasma (CP) is a therapeutic option for these patients. Our objective is to retrospectively evaluate the efficacy and adverse events of CP treatment in this population group.

Methods: Forty one patients over 80 years old with COVID-19 pneumonia received CP added to standard treatment, $51.2 \%$ with high anti-SARS-CoV-2 lgG titers and $48.8 \%$ with low titers. Median time between the onset of symptoms and the infusion of plasma was 7 days (IQR 4-10). A similar group of 82 patients who received only standard treatment, during a period in which CP was not available, were selected as a control group.

Results: In-hospital mortality was $26.8 \%$ for controls and $14.6 \%$ for CP patients ( $P=0.131)$ and ICU admission was 8.5\% for controls and $4.9 \%$ for $C P$ patients $(P=0.467)$. Mortality tended to be lower in the high-titer group (9.5\%) than in the low-titer group (20\%), and in patients transfused within the first 7 days of symptom onset (10\%) than in patients transfused later (19.1\%), although the differences were not statistically significant $(P=0.307$ and $P=0.355$ respectively). There was no difference in the length of hospitalization. No significant adverse events were associated with CP treatment.

Conclusions: Convalescent plasma treatment in patients over 80 years old with COVID-19 pneumonia was well tolerated but did not present a statistically significant difference in hospital mortality, ICU admission, or length of hospitalization. The results should be interpreted with caution as only half the patients received high-titer CP and the small number of patients included in the study limits the statistical power to detect significant differences.
\end{abstract}

Trial registration: CEIm Cantabria \# 2020.127.

Keywords: COVID-19, Convalescent plasma, Older adults, Transfusion

\section{Background}

The COVID-19 pandemic has created an unprecedented clinical situation in which patients over 65 years of age are more severely affected [1-3]. Chronic conditions, such as cardiovascular diseases, hypertension, obesity,

\footnotetext{
* Correspondence: joseinigo.romon@scsalud.es

${ }^{1}$ Hematology and Hemotherapy Service, Hospital Universitario Marqués de Valdecilla, Avenida Valdecilla, s/n, 39008 Santander, Spain

Full list of author information is available at the end of the article
}

cognitive impairment or diabetes are major risk factors for mortality in patients with COVID-19 [1, 3, 4]. Residing in long term care facilities (LTCF), where older people with multiple comorbidities live in close contact, facilitating virus transmission, can also be a risk [5].

Older patients, over 80 years of age, often residing in LTCF and affected by multiple comorbidities, have suffered the highest mortality rate, ranging from 15 to over $50 \%[1-3,5-7]$. In fact, the country-specific case fatality

(c) The Author(s). 2021 Open Access This article is licensed under a Creative Commons Attribution 4.0 International License, which permits use, sharing, adaptation, distribution and reproduction in any medium or format, as long as you give appropriate credit to the original author(s) and the source, provide a link to the Creative Commons licence, and indicate if changes were made. The images or other third party material in this article are included in the article's Creative Commons licence, unless indicated otherwise in a credit line to the material. If material is not included in the article's Creative Commons licence and your intended use is not permitted by statutory regulation or exceeds the permitted use, you will need to obtain permission directly from the copyright holder. To view a copy of this licence, visit http://creativecommons.org/licenses/by/4.0/. The Creative Commons Public Domain Dedication waiver (http//creativecommons.org/publicdomain/zero/1.0/) applies to the data made available in this article, unless otherwise stated in a credit line to the data. 
rate is predominantly determined by the proportion of older individuals affected [8]. Accordingly, in Europe, where life expectancy usually exceeds 80 years, COVID19 infection has had a strong impact with high mortality.

In addition, older patients are frequently not candidates for admission to Intensive Care Units (ICU), due to their multiple comorbidities and their high mortality rate, reaching $70-80 \%[6,9]$.

COVID-19 convalescent plasma (CP) is a source of antiviral neutralizing antibodies [10] and has been used to treat hospitalized patients.

The US Food and Drug Administration (FDA), given the lack of effective treatments, granted CP Emergency Use Authorization. Conditions have recently been revised and an early administration of high-titer $\mathrm{CP}$ is currently required [11]. This strategy was also implemented by the European Union [12].

In 2020, a CP program was started for the treatment of COVID-19 patients with pneumonia in our region. In this paper we report the results of an interim analysis of the program's development, focusing on patients over 80 years old, the population group with the highest COVID-19 induced mortality in the region.

\section{Methods}

This study analyses the effect of $\mathrm{CP}$ administration to hospitalized adults over 80 years old with pneumonia and compares the results against a control group receiving standard treatment.

\section{Convalescent plasma treatment protocol}

In 2020 , a CP prospective study program promoted by the Regional Health Service was started for the treatment of COVID-19 patients with pneumonia recruited at the three regional hospitals (one academic and two community hospitals). Authorization from the Medical Ethics Committee was obtained.

The use of $\mathrm{CP}$ had previously been authorized by the National Council for Transfusion Safety [13] and the European Commission [12]. The use of $\mathrm{CP}$ was authorized under the provision that a strict vigilance of adverse events and of clinical results was to be maintained. Hospitals and Blood collection centers could manage their own donor base of convalescent patients, and guarantee that donations had COVID-19 antibodies, giving always donations with the highest titers available. A serum sample of all donations had to be kept for future analysis. To ensure that, all patients had to be prospectively registered on an anonymized database managed by the Spanish Government, that would in turn transfer it to the European Commission. Data regarding the patients' demographic data, most relevant comorbidities, identification of transfused units and follow up data up to 30 days after transfusion were collected.

Adult patients were treated at dedicated COVID-19 wards, irrespective of their age. CP treatment was optional for all adult COVID-19 patients with radiologically confirmed pneumonia, according to the criteria of the patient's physician, and was compatible with the administration of standard treatment. Informed consent was obtained from the patient or their legal representative. Patients in the ICU were only given plasma during their first day at the ICU. Exclusion criteria were IgA deficiency, known severe adverse reactions to plasma transfusion, and refusal to consent.

The standard of care consisted of steroids (after 7 days of symptoms) and prophylactic heparin. Remdesivir was administered within the first 7 days of symptom onset, and tocilizumab was administered with raised IL-6 levels. Oxygen, antibiotics and other medical treatments were given as required. Criteria for ICU admittance were the patient's performance status and a life expectancy of at least 6 months before COVID, not their age as such.

$\mathrm{CP}$ treatment consisted of one $300 \mathrm{~mL} \mathrm{ABO}$ compatible plasma unit administered to each patient. Special attention was paid to the avoidance of fluid overload.

To ensure a uniform implementation of the protocol, the treatment protocol was approved by the Heads of Departments of Infectious Diseases, Internal Medicine, Pneumology and Intensive Care of the hospitals treating COVID patients. Periodical follow up meetings between the treating physicians and the steering hematological team were held.

\section{Convalescent plasma donations}

Plasma donors had to be asymptomatic for at least 14 days after COVID-19 infection prior to donation. Prospective donors complied with the legal donor selection criteria for blood donation.

To prevent transfusion-associated acute lung injury (TRALI), which could worsen or mimic COVID-19 lung damage, plasma donors with previous pregnancies or transfusions, at risk of developing anti-HLA antibodies known to cause TRALI, were screened and excluded when positive.

Donations were initially tested by qualitative enzymelinked immunosorbent assay (ELISA) for anti-SARS$\mathrm{CoV}-2$ antibodies. Subsequently, all donations were prospectively tested using the VITROS anti SARS-CoV2 IgG antibody test (Ortho-Clinical Diagnostics), and stored serum samples were retrospectively retested. CP units were classified as high-titer and low-titer according to FDA recommendations [11]. As a result, some donations used during the first weeks of the study were tested retrospectively, this revealed that some patients treated during the first weeks of the study randomly received 
$\mathrm{CP}$ units with lower titers. In a small group of patients, lower-titer $\mathrm{CP}$ was given by order of the treating physicians when no other option was available. This possibility had been contemplated by the Directive issued by the National Council for Transfusion Safety [13].

\section{Patients and controls}

For an interim analysis of the study, we analyzed the subgroup of 41 patients over 80 years old, treated with CP between August and December 2020, chosen consecutively from those admitted to hospital during that period. As a control group, we chose a group of $82 \mathrm{pa}-$ tients within the same age range, admitted to hospital during a 6-week period between September and November 2020, when CP was unavailable due to a breach in stock. Patients who died within the first 3 days after admission were excluded as controls. We did not extend the analysis beyond January because of the predominance of the British COVID-19 variant, which was considered a different clinical setting.

All patients had a positive RT-PCR test for SARSCoV-2 in a nasopharyngeal sample and a compatible chest radiography assessed by a trained radiologist.

Data extracted from the patients' records included age, sex, residence (home vs. LTCF), comorbidities and arterial oxygen saturation, $\mathrm{SaO} 2 / \mathrm{FiO} 2(\mathrm{SAFI})$ and $\mathrm{PaO} 2 / \mathrm{FiO} 2$ (PAFI that had been recorded upon hospital admission. Before transfusion, patients in the $\mathrm{CP}$ group were assessed via the WHO scale for the improvement of COVID-19, [14] and assessment in the control group took place at the time corresponding to the median number of days between admission and plasma infusion in the CP group. Pharmacological treatment for COVID19 pneumonia was also recorded.

\section{Outcomes}

The primary outcome was the impact of $\mathrm{CP}$ administration on all cause in-hospital mortality rate. Secondary outcomes were the need for ICU admission and length of hospital stay. Adverse events within $24 \mathrm{~h}$ of transfusion were also recorded.

\section{Statistical analysis}

Data were recorded on a Microsoft Excel 2008 database (Redmond, Washington, USA) and analysed using the StataIC-16 (College Station, Texas, USA) for the descriptive and the statistical inference, and SPSS Statistics 25 (Brussel, Belgium) application for the survival analysis. Normality, asymmetry and skewness were analyzed with the Shapiro-Wilk normality test and the normal standardized probability plot. Quantitative variables were analyzed by comparing their means with the t-test. Categorical variables were analyzed with Pearson's chisquared if samples had a relative frequency higher than five or the Fisher's exact test if their relative frequency was lower. Survival analysis was performed using the Kaplan-Meier method. Statistical significance was achieved if $p$-value $\leq 0.05$.

For a multivariate analysis, we performed a Cox proportional hazards model analysis to investigate the influence of convalescent plasma treatment on mortality.

\section{Results}

A total of 123 patients over 80 years of age were analyzed (mean age 86.2, SD 4.6), 41 in the CP group and 82 in the control group. Women accounted for $41 \%$ of patients (CP 53.7\% vs. controls 35.4\%) Patients living in LTCF accounted for $20.3 \%$, and the most prevalent comorbidities were hypertension (78.1\%), cardiovascular disease (51.2\%), dyslipidemia (48.0\%), cognitive impairment $(31.7 \%)$ and diabetes (30.9\%), with no differences between groups (Table 1$)$.

Median time from the onset of symptoms to hospital admission (5 days, IQR 2-7), arterial oxygen saturation, SAFI and PAFI at admission, and WHO scale for COVID pneumonia assessment were comparable between both groups (Table 1).

Most patients in both arms received anticoagulants, antibiotics, and glucocorticoids, and less frequently Tocilizumab, Anakinra or Lopinavir/Ritonavir, with no differences between groups. However, Remdesivir was administered more often to patients in the plasma group (CP 31.7\%, vs. controls 6.1\%; $P<0.001$ ).

Median time from the onset of symptoms and hospital admission to CP administration was 7 (IQR 4-10) and 1 (IQR 0-2) days, respectively. High-titer units were given to 21 patients $(51.2 \%)$ and low-titer $\mathrm{CP}$ to 20 cases (48.8\%).

Two mild transfusion adverse events $(4.9 \%)$ were reported, one patient complained of headache and another had a fever after transfusion, both responding to symptomatic treatment.

\section{Clinical outcome}

The all-cause in-hospital mortality (IHM) rate was $22.8 \%$ (28 patients) while $77.2 \%$ (95 patients) were discharged. Nine patients $(7.3 \%)$ were admitted to ICU for mechanical ventilation, five of which died (55.6\%). Patients who were not considered candidates for ICU admission received appropriate palliative care where necessary.

The length of stay for discharged patients was 11 days (IQR 9-16), with no differences between CP and control cohorts.

Although the CP group presented a lower IHM rate (CP 14.6\% vs. controls 26.8\%) and lower ICU admission (CP 4.9\% vs. control 8.5\%), these differences were not statistically significant $(P=0.131$ and $P=$ 
Table 1 Demographic characteristics, comorbidities, clinical data, treatment and outcomes of the overall sample and each cohort

\begin{tabular}{|c|c|c|c|c|}
\hline & $\begin{array}{l}\text { Overall Cohort } \\
(n=123)\end{array}$ & $\begin{array}{l}\text { Convalescent Plasma } \\
(n=41)\end{array}$ & $\begin{array}{l}\text { Control Group } \\
(n=82)\end{array}$ & $p$-value \\
\hline Age, mean (SD) & $86.2(4.60)$ & $86.7(5.02)$ & $85.9(4.39)$ & 0.416 \\
\hline Female sex, n (\%) & $51(41.5)$ & $22(53.7)$ & $29(35.4)$ & 0.052 \\
\hline \multicolumn{5}{|l|}{ Comorbidity } \\
\hline Mental impairment, n (\%) & $39(31.7)$ & $14(34.2)$ & $25(30.5)$ & 0.681 \\
\hline Hypertension, n (\%) & $96(78.1)$ & $32(78.1)$ & $64(78.1)$ & 1.000 \\
\hline Diabetes mellitus, n (\%) & $38(30.9)$ & $9(22.0)$ & $29(35.4)$ & 0.129 \\
\hline Dyslipidemia, n (\%) & $59(48.0)$ & $17(41.5)$ & $42(51.2)$ & 0.307 \\
\hline Obesity, n (\%) & $19(15.5)$ & $3(7.3)$ & $16(19.5)$ & 0.078 \\
\hline Cardiovascular disease, n (\%) & $63(51.2)$ & $18(43.9)$ & $45(54.9)$ & 0.251 \\
\hline CPD, n (\%) & $28(22.8)$ & $8(19.5)$ & $20(24.4)$ & 0.543 \\
\hline Current or past smoker, n (\%) & $24(19.5)$ & $8(19.5)$ & $16(19.5)$ & 1.000 \\
\hline Chronic kidney disease, n (\%) & $25(20.3)$ & $7(17.1)$ & $18(22.0)$ & 0.526 \\
\hline Cancer, n (\%) & $34(27.6)$ & $13(31.7)$ & $21(25.6)$ & 0.476 \\
\hline LTCF residents, n (\%) & $25(20.3)$ & $8(19.5)$ & $17(20.7)$ & 0.874 \\
\hline Days from symptom onset to admission $\left(^{*}\right)$ & $5(2-7)$ & $5(2-7)$ & $5(3-7)$ & 0.877 \\
\hline Days from symptom onset to CP infusion $\left(^{*}\right)$ & N/A & $7(4-10)$ & N/A & - \\
\hline Days from admission to CP infusion $(*)$ & N/A & $1(0-2)$ & N/A & - \\
\hline $\mathrm{SaO}_{2}(\mathrm{mmHg})$ at admission $(+)$ & $91.8(0.42)$ & $92.1(0.60)$ & $91.7(0.56)$ & 0.606 \\
\hline Estimated $\mathrm{PaO}_{2} / \mathrm{FiO}_{2}$ at admission (+) & $321.5(5.70)$ & $319.9(8.83)$ & $322.3(7.35)$ & 0.848 \\
\hline WHO scale $(+)$ & $3.8(0.06)$ & $3.8(0.09)$ & $3.8(0.07)$ & 1.000 \\
\hline \multicolumn{5}{|l|}{ Treatment, n (\%) } \\
\hline Anticoagulants & $122(99.2)$ & $41(100)$ & $81(98.8)$ & 0.667 \\
\hline Antibiotics & $119(96.8)$ & $39(95.1)$ & $80(97.6)$ & 0.472 \\
\hline Glucocorticoids & $100(81.3)$ & $32(78.1)$ & $68(82.9)$ & 0.513 \\
\hline Remdesivir & $18(14.6)$ & $13(31.7)$ & $5(6.1)$ & 0.000 \\
\hline Tocilizumab & $14(11.4)$ & $7(17.1)$ & $7(8.5)$ & 0.160 \\
\hline Anakinra & $2(1.6)$ & $1(2.4)$ & $1(1.2)$ & 0.557 \\
\hline Lopinavir/ritonavir & $1(0.8)$ & $1(2.4)$ & $0(0.0)$ & 0.333 \\
\hline \multicolumn{5}{|l|}{ Outcomes } \\
\hline In-hospital mortality, n (\%) & $28(22.7)$ & $6(14.6)$ & $22(26.8)$ & 0.131 \\
\hline ICU admission, $\mathrm{n}(\%)$ & $9(7.3)$ & $2(4.9)$ & $7(8.5)$ & 0.467 \\
\hline LoS, discharged, median (IQR) & $11(9-16)$ & $11(9-16)$ & $11(7.5-16)$ & 0.073 \\
\hline LoS, dead, median (IQR) & $9(6-12)$ & $11(7-22)$ & $8.5(5-12)$ & 0.067 \\
\hline
\end{tabular}

SD Standard Deviation, CPD Chronic Pulmonary Disease, LTCF Long Term Care Facilities, SaO2 Arterial oxygen saturation, FiO2 Fraction of inspired Oxygen, N/A Not Applicable, ICU Intensive Care Unit, LoS Length of Stay, IQR Interquartile Range

(*) Expressed as median (Interquartile Range). (+) Expressed as mean (Standard Deviation)

0.467, Overall survival: chi-squared log-Rank: 3.79 , $P=0.052)$ (Table 1).

There was a lower mortality trend for the subgroup of 21 patients who received high-titer $\mathrm{CP}$ (9.5\%) and for the 20 patients who received CP within 6 days of symptom onset $(10 \%)$, but these differences were not significant (log-Rank: 4.23, $P=0.121$ and $P=0.355$ respectively) (Table 2 and Fig. 1).
In a multivariate model using the Cox proportional hazards model, the HR for the influence of $\mathrm{CP}$, adjusted by age, WHO initial status, sex and ICU admittance was $0.887(p=0.560)$.

Acute respiratory distress syndrome and subsequent multi-organ failure was the most common cause of death (92.9\%). One patient from each group died of retroperitoneal hemorrhage. 
Table 2 In-hospital mortality related to groups, CP titers and days from symptom onset

\begin{tabular}{llll}
\hline & Sample Size, $\mathbf{n}$ & $\begin{array}{l}\text { Mortality, } \\
\mathbf{n}(\%)\end{array}$ & $\boldsymbol{p}$-value \\
\hline Control group & 82 & $22(26.8)$ & 0.128 \\
Convalescent Plasma group & 41 & $6(14.6)$ & \\
Low-titer CP & 20 & $4(20.0)$ & 0.307 \\
High-titer CP & 21 & $2(9.5)$ & \\
Symptom onset to CP $<7$ days & 20 & $2(10.0)$ & 0.355 \\
Symptom onset to CP $\geq 7$ days & 21 & $4(19.1)$ & \\
\hline
\end{tabular}

$C P$ Convalescent Plasma

Only one patient in the control group was diagnosed with thromboembolic disease (already present at hospital admission).

\section{Discussion}

We describe the results of $\mathrm{CP}$ as adjuvant to standard treatment for COVID-19 infection with pneumonia in a group of 41 patients over 80 years of age with multiple comorbidities, and compare them with a control group of 82 similar patients.

Most deaths in both groups were directly attributed to COVID-19 infection. Although patients treated with $\mathrm{CP}$ had lower overall in-hospital mortality than controls (14.6\% vs. 26.8\%) and lower ICU admissions $(4.9 \%$ vs. $8.5 \%)$ the difference was not statistically significant. Similar to other reports $[6,9]$, the mortality of patients requiring ICU care was very high (55.6\%).

The results of studies on CP treatment present apparently discrepant conclusions.

In two randomized studies, the administration of $\mathrm{CP}$ in hospitalized patients with pneumonia did not show a better clinical evolution or decrease in mortality $[15,16]$. In contrast, a recent randomized study shows that early administration of $\mathrm{CP}$ (within $72 \mathrm{~h}$ after the onset of symptoms) with high anti-SARS-CoV-2 levels, significantly reduces the progression to severe respiratory disease [17]. Also, a retrospective study of 3082 cases showed that mortality was lower in transfused patients with high titers of anti-SARS-CoV-2 IgG antibodies than in those receiving low-titer $\mathrm{CP}$, with no benefit for patients on mechanical ventilation at the time of transfusion [18]. Similarly, another report describes a significant decrease in mortality in patients receiving high-titer CP within the first $72 \mathrm{~h}$ after admission [19]. Results were recently updated establishing that the optimal approach to reduce mortality appears to be the transfusion of high-titer CP within the first $44 \mathrm{~h}$ after hospitalization [20].

Discrepancies may be the result of the heterogeneity of the patients selected (time of evolution and severity of the disease) and in the antibody levels of the CP administered.

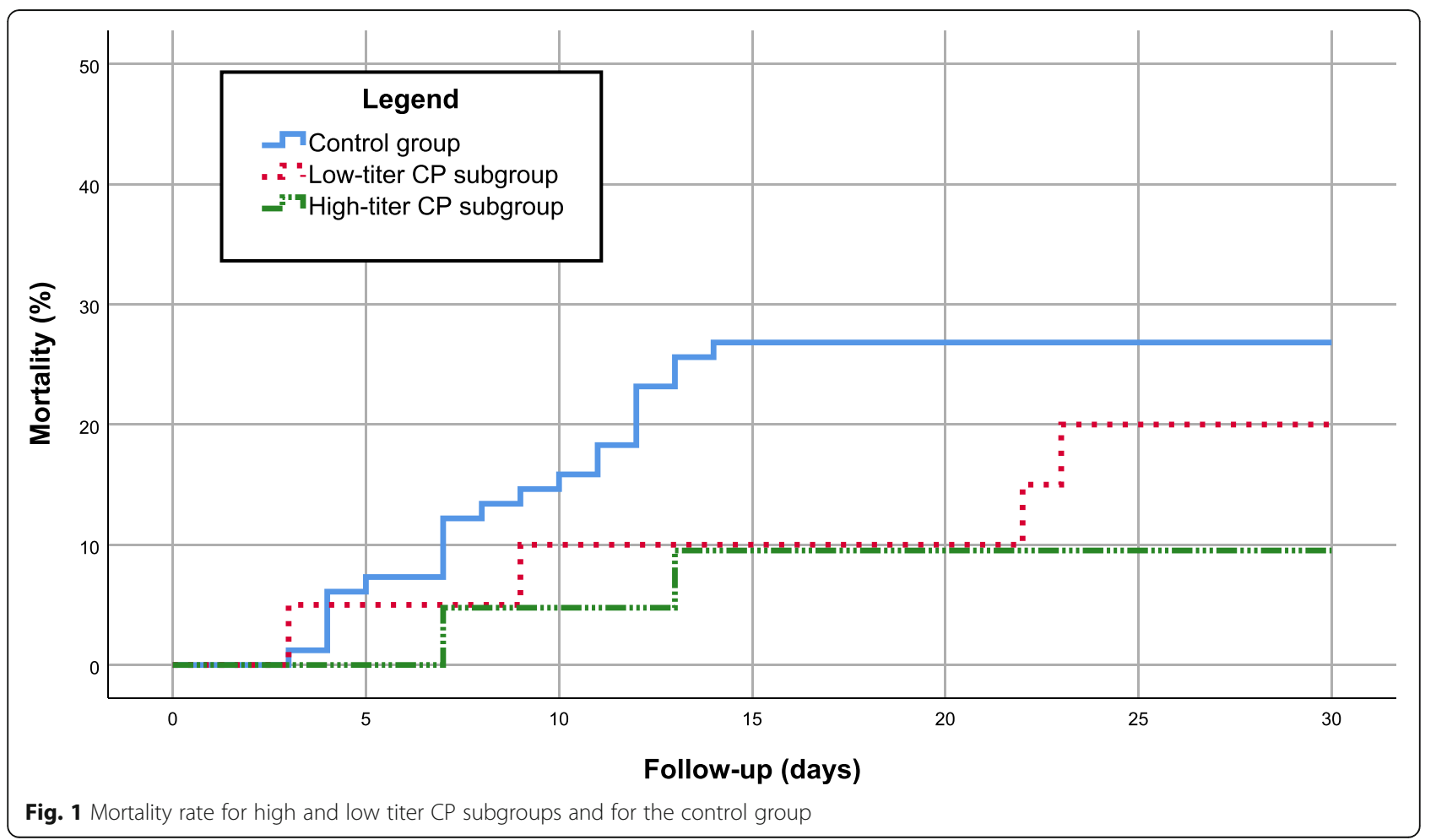


Our results suggest that $\mathrm{CP}$ treatment in this population group does not improve clinical results. However, the absence of statistically significant differences in favor of CP may be due to several factors. First, lack of statistical power because of the small number of patients treated. Second, the late administration of convalescent plasma (median 7 days after the onset of symptoms), as the subgroup of patients receiving CP treatment within 7 days of symptoms had a mortality of $10 \%$, compared with $19 \%$ for those who received it later, and $26 \%$ for controls. Third, only half of the patients received hightiter CP with a $9.5 \%$ mortality, compared with $20 \%$ for patients receiving low-titer $\mathrm{CP}$ and $26.8 \%$ for controls. Finally, we cannot rule out that $\mathrm{CP}$ treatment could be ineffective.

A recent report explored the use of $\mathrm{CP}$ in 22 older patients living in LTCF, and the overall mortality compared favorably with the Health authorities-reported mortality in LTCF in the same region and period of time [21]. The researchers admit that, given the emergency situation, some of their patients died in their LTCF without receiving adequate treatment or supportive care in a hospital setting. Therefore, it is likely that the control group, even if it had similar baseline characteristics, did not receive the same treatment in the different LTCFs, which may explain the better results in the group treated with CP. In our case, both patients and controls were treated at the same hospitals, by the same professional teams, with homogeneous criteria. In our region there have been no restrictions for patients' access to hospital or ICU. Some of Franchini's patients received two or three plasma units, while ours received only one. Moreover, Franchini's patients had lower obesity, cardiovascular and chronic kidney disease rates than ours.

$\mathrm{CP}$ administration was well tolerated, with mild adverse events in $4.9 \%$ of the patients, in agreement with a large series in which the incidence of serious transfusion reactions was under 1\% [22]. The incidence of thrombotic events was very low, probably because most patients received anticoagulant drugs, usually in prophylactic doses.

Although our study could not demonstrate the effectivity of CP treatment, $\mathrm{CP}$ can still play an important role in the treatment of selected patients with impaired immune response [23]. Considering the low incidence of $\mathrm{CP}$ adverse events and the scarce therapeutic alternatives for this group of older, high-risk patients, it is worth conducting further studies. To optimize treatment, CP must contain high anti-SARS-CoV-2 titers and should be administered early in the course of the disease.

Our study has some limitations. 1) Being a retrospective study, some information may have been lost if it was not reflected in medical records, such as the existence of some comorbidities. 2) The onset of symptoms may have occurred before the recorded date, since many of these patients, with cognitive impairment, lack the capacity to express themselves and the symptoms may have gone unnoticed. 3) The non-randomized design of the study may have favored a bias in the selection of patients who received $\mathrm{CP}$, but we believe the controls are reasonably well chosen, as they were taken from a period when $\mathrm{CP}$ could not be obtained due to a breach in stock. 4) Patients in the treatment group received remdesivir more often than controls, and this could have improved their outcome. Remdesivir was only administered within 7 days of symptom onset, and in both groups the time from onset of symptoms to hospital admittance was similar (Control 5.2 days vs. Plasma 5.3 days; $p=0.877$ ). Thus, we can only speculate that physicians considered that these patients were in worse condition and decided to use both $\mathrm{CP}$ and remdesivir. 5) Our patients were treated at dedicated COVID units, but lacked a specific geriatric assessment, that could have helped to reach more informed conclusions.

\section{Conclusion}

$\mathrm{CP}$ added to standard therapy in patients over 80 years of age did not significantly reduce overall in-hospital mortality, ICU admission or time to hospital discharge, compared with standard treatment alone. Interpretation is limited by the small sample size and the high percentage of plasma units with low-titer antibodies.

\section{Abbreviations \\ CP: Convalescent plasma; ICU: Intensive care unit; LoS: Length of stay; LTCF: Long term care facilities; FDA: US Food and Drug Administration; TRALI: Transfusion-associated acute lung injury; ELISA: Enzyme linked immunosorbent assay; IHM: In-hospital mortality}

\section{Acknowledgements}

The authors would like to thank María Abando and Alberto Vazquez for their unconditional support and continuous help in curating data and contacting patients for this work.

Authors' contributions

IR edited the different drafts. MB designed the study, wrote the drafts and oversaw the process. JJD performed the statistical analysis, tables and graphics. JLA provided plasma donors. All the authors collected clinical data, reviewed and approved the subsequently draft versions.

\section{Funding}

The authors have received no compensation or pay, financial or otherwise to prepare this paper from any public, commercial or not-for-profit entities.

\section{Availability of data and materials}

The datasets used and analyzed during the current study are available from the corresponding author on reasonable request.

\section{Declarations}

Ethics approval and consent to participate

This work received the approval of the regional Ethics Committee [Comité de Ética de la Investigación con Medicamentos de Cantabria (CEIm de Cantabria). IDIVAL, Edificio IDIVAL, 3a Planta, Avda. Cardenal Herrera Oria s/n, 39011 Santander, Spain. eclinicos1@idival.org.] 
All patients gave informed consent to be treated with convalescent plasma and their data to be used for this research. Spanish legislation on patients' privacy and self-determination allows verbal consent in emergency situations, provided the physician gives the patient enough information and records the terms of consent on the patient's medical record. The Ethics Committee accepted the pandemic and associated measures (patients' isolation, lockdowns) could be considered an emergency. Due to the isolation mandated for COVID patients, the electronic pads used sign the consent documents could not be brought into the patients' rooms, and consent had to be given verbally and registered by the physician in the patients' medical records. If patients could not express consent, their legal representatives were contacted. This could also be done by telephone, as strict lockdown rules often impeded relatives or tutors visiting hospital.

All methods were performed in accordance with the relevant guidelines and regulations, following the Declaration of Helsinki for the ethical principles for medical research involving human subjects, including research on identifiable human material and data.

\section{Consent for publication}

Not applicable.

\section{Competing interests}

The authors declare that they have no competing interests.

\section{Author details}

${ }^{1}$ Hematology and Hemotherapy Service, Hospital Universitario Marqués de Valdecilla, Avenida Valdecilla, s/n, 39008 Santander, Spain. ${ }^{2}$ Banco de Sangre y Tejidos de Cantabria, 39121 Liencres, Spain. ${ }^{3}$ Intensive Care Service, Hospital Universitario Marqués de Valdecilla, 39008 Santander, Spain. ${ }^{4}$ Infectious Diseases Service, Hospital Universitario Marqués de Valdecilla, 39008 Santander, Spain. ${ }^{5}$ Pneumology Service, Hospital Universitario Marqués de Valdecilla, 39008 Santander, Spain. Internal Medicine Service, Hospital Universitario Marqués de Valdecilla, 39008 Santander, Spain. ${ }^{7}$ Haematology Service, Hospital de Sierrallana, 39300 Torrelavega, Spain. ${ }^{8}$ Internal Medicine Service, Hospital de Sierrallana, 39300 Torrelavega, Spain. ${ }^{9}$ Internal Medicine Service, Hospital de Laredo, 39770 Laredo, Spain.

Received: 24 March 2021 Accepted: 30 August 2021

Published online: 18 October 2021

\section{References}

1. Wu Z, McGoogan JM. Characteristics of and important lessons from the coronavirus disease 2019 (COVID-19) outbreak in China: summary of a report of 72314 cases from the Chinese Center for Disease Control and Prevention. JAMA. 2020 Apr;323(13):1239-42. https://doi.org/10.1001/jama.2 020.2648 .

2. Niu S, Tian S, Lou J, Kang X, Zhang L, Lian H, et al. Clinical characteristics of older patients infected with COVID-19: a descriptive study. Arch Gerontol Geriatr. 2020 Jul;89:104058. https://doi.org/10.1016/j.archger.2020.104058.

3. Becerra-Muñoz VM, Núñez-Gil IJ, Eid CM, García Aguado M, Romero R, Huang J, et al. Clinical profile and predictors of in-hospital mortality among older patients hospitalised for COVID-19. Age Ageing. 2021;50(2):326-34. https://doi.org/10.1093/ageing/afaa258.

4. Wang B, Li R, Lu Z, Huang Y. Does comorbidity increase the risk of patients with COVID-19: evidence from meta-analysis. Aging (Albany NY). 2020 Apr; 12(7):6049-57. https://doi.org/10.18632/aging.103000.

5. D'ascanio M, Innammorato M, Pasquariello L, Pizzirusso D, Guerrieri G, Castelli S, et al. Age is not the only risk factor in COVID-19: the role of comorbidities and of long staying in residential care homes. BMC Geriatr. 2021;21(1):63 Available from: https://bmcgeriatr.biomedcentral.com/a rticles/10.1186/s12877-021-02013-3.

6. Nabors C, Sridhar A, Hooda U, Lobo SA, Levine A, Frishman WH, et al. Characteristics and outcomes of patients 80 years and older hospitalized with coronavirus disease 2019 (COVID-19). Cardiol Rev. 2021;29(1):39-42. https://doi.org/10.1097/CRD.0000000000000368.

7. Vrillon A, Hourregue C, Azuar J, Grosset L, Boutelier A, Tan S, et al. COVID-19 in older adults: a series of 76 patients aged 85 years and older with COVID -19. J Am Geriatr Soc. 2020;68(12):2735-43. https://doi.org/10.1111/jgs.16894.
8. Hoffmann C, Wolf E. Older age groups and country-specific case fatality rates of COVID-19 in Europe, USA and Canada. Infection. 2021;49(1):111-6. https://doi.org/10.1007/s15010-020-01538-w.

9. Lim ZJ, Subramaniam A, Ponnapa Reddy M, Blecher G, Kadam U, Afroz A, et al. Case fatality rates for patients with COVID-19 requiring invasive mechanical ventilation. A Meta-analysis. Am J Respir Crit Care Med. 2021; 203(1):54-66. https://doi.org/10.1164/rccm.202006-2405OC.

10. Casadevall A, Pirofski L. The convalescent sera option for containing COVID19. J Clin Invest. 2020;130(4):1545-8. https://doi.org/10.1172/JCl138003.

11. Villa CH. CLINICAL MEMORANDUM. COVID-19 Convalescent Plasma. EUA 26382: Food and Drug Administration. https://www.fda.gov/media/141480/ download. Accessed 17 Sept 2021.

12. COVID-19 Convalescent Plasma Transfusion | Public Health. [cited 2021 Feb 7]. Available from: https://ec.europa.eu/health/blood_tissues_organs/covid-1 9 en

13. Moro E. DIRECTRICES PARA LA OBTENCIÓN DE PLASMA DE DONANTES CONVALECIENTES DE LA COVID-19 Comité Científico para la Seguridad Transfusional (CCST). Madrid; 2020. [cited 2021 Feb 2]. Available from: https://www.mscbs.gob.es/profesionales/saludPublica/medicinaTransfusiona l/acuerdos/docs/COVID-19_Directrices_Plasma_donantes_convalecientes.pdf

14. WHO R\&D Blueprint novel Coronavirus COVID-19 Therapeutic Trial Synopsis. 2020. https://cdn.who.int/media/docs/default-source/blue-print/covid-19therapeutic-trial-synopsis.pdf?sfvrsn=44b83344_1\&download=true. Accessed 17 Sept 2021.

15. Agarwal A, Mukherjee A, Kumar G, Chatterjee P, Bhatnagar T, Malhotra P, et al. Convalescent plasma in the management of moderate covid-19 in adults in India: open label phase II multicentre randomised controlled trial (PLACID Trial). BMJ. 2020;371:m3939.

16. Simonovich VA, Burgos Pratx LD, Scibona P, Beruto MV, Vallone MG, Vázquez C, et al. A randomized trial of convalescent plasma in Covid-19 severe pneumonia. N Engl J Med. 2020; [cited 2021 Feb 2]; Available from: https://www.nejm.org/doi/full/10.1056/NEJMoa2031304.

17. Libster R, Pérez Marc G, Wappner D, Coviello S, Bianchi A, Braem V, et al. Early high-titer plasma therapy to prevent severe Covid-19 in older adults. N Engl J Med. 2021:NEJMoa2033700 [cited 2021 Feb 2]. Available from: https://www.nejm.org/doi/full/10.1056/NEJMoa2033700.

18. Joyner MJ, Carter RE, Senefeld JW, Klassen SA, Mills JR, Johnson PW, et al. Convalescent plasma antibody levels and the risk of death from Covid-19. N Engl J Med. 2021:NEJMoa2031893 [cited 2021 Feb 2]. Available from: https://www.nejm.org/doi/full/10.1056/NEJMoa2031893.

19. Salazar E, Christensen PA, Graviss EA, Nguyen DT, Castillo B, Chen J, et al. Treatment of Coronavirus Disease 2019 patients with convalescent plasma reveals a signal of significantly decreased mortality. Am J Pathol. 2020; 190(11):2290-303 [cited 2021 Feb 2]. Available from: https://pubmed.ncbi. nlm.nih.gov/32795424/.

20. Salazar E, Christensen PA, Graviss EA, Nguyen DT, Castillo B, Chen J, et al. Significantly decreased mortality in a large cohort of coronavirus disease 2019 (COVID-19) patients transfused early with convalescent plasma containing high-titer anti-severe acute respiratory syndrome coronavirus 2 (SARS-CoV-2) spike protein IgG. Am J Pathol. 2021;191(1):90-107. https://doi. org/10.1016/j.ajpath.2020.10.008.

21. Franchini M, Glingani C, Morandi M, Corghi G, Cerzosimo S, Beduzzi G, et al. Safety and efficacy of convalescent plasma in elderly COVID-19 patients: the RESCUE Trial. Mayo Clin Proc Innov Qual Outcomes. 2021;5(2):403-12 [cited 2021 May 11]. Available from: https://doi.org/10.1016/j.mayocpiqo.2021.01. 010.

22. Joyner MJ, Bruno KA, Klassen SA, Kunze KL, Johnson PW, Lesser ER, et al. Safety update: COVID-19 convalescent plasma in 20,000 hospitalized patients. Mayo Clin Proc. 2020 Sep;95(9):1888-97. https://doi.org/10.1016/j. mayocp.2020.06.028

23. Hueso T, Pouderoux C, Péré H, Beaumont A, Raillon L-A, Ader F, et al. Convalescent plasma therapy for B-cell-depleted patients with protracted COVID-19. Blood. 2020;136(20):2290-5. Available from: https://ashpublica tions.org/blood/article/136/20/2290/463806/Convalescent-plasma-therapyfor-Bcelldepleted. https://doi.org/10.1182/blood.2020008423.

\section{Publisher's Note}

Springer Nature remains neutral with regard to jurisdictional claims in published maps and institutional affiliations. 